\title{
Noninsured services provided with insured cataract surgery in Canada: ensuring transparent and fair treatment for patients
}

\author{
Chryssa N. McAlister MD, lqbal Ike K. Ahmed MD
}

CMAJ Podcast: author interview at soundcloud.com/cmajpodcasts/mcalister-cataract

$\longrightarrow$ ataract surgery is the most commonly performed medically necessary procedure in Canada, and the costs are covered under provincial and territorial health insurance plans. Patients who require this surgery are frequently offered one or more of a number of optional services related to refractive correction during cataract surgery. These services, which include innovative diagnostics, laser therapies and surgical devices, are supplied at additional cost to the patient because they are not deemed to be medically necessary by government funding agencies across Canada. These noninsured technologies may allow for more predictable refractive results, freedom from the need for corrective lenses, and enhanced quality of vision over and above the benefits of cataract removal, and they are often provided in conjunction with publicly insured cataract surgery.

Ophthalmologists who provide noninsured services during insured surgical procedures must consider specific ethical challenges. These challenges may also apply to other specialties in which noninsured services are routinely provided during insured consultations or procedures. We discuss these ethical considerations and propose a framework for ensuring that patients are properly and fairly informed about their choices for surgery.

\section{Which ophthalmologic treatments are not publicly funded and why?}

Technological advances in cataract surgery adopted by publicly funded insurance plans over the past few decades, such as phacoemulsification, have revolutionized the procedure and allowed for faster and safer surgery, and better surgical outcomes. ${ }^{1}$ Newer ophthalmic innovations in cataract surgery aim to enhance refractive outcomes for patients interested in independence from corrective lenses and are not currently covered by public insurance plans. Examples include aspheric, multifocal and accommodating intraocular lenses (specialfeature IOLs); astigmatism management; diagnostics, such as optical biometry; and imageguided femtosecond laser-assisted cataract surgery. Despite the impressive current standard of care for cataract surgery, these innovations may additionally enhance refractive outcomes ${ }^{2}$ and reduce a patient's need for corrective lenses — but at a financial cost.

Refractive correction with glasses, contact lenses or laser refractive surgery is not covered by publicly funded health plans, and because the optional noninsured services performed at the time of cataract surgery improve refractive outcomes rather than reduce complication rates of surgery, public coverage is deemed unwarranted. For example, a 2009 analysis by Health Quality Ontario concluded that special-feature IOLs are convenience items that are not medically necessary. ${ }^{3}$ The current yearly cost of providing IOLs to Ontario patients is about $\$ 27.4$ million for standard lenses. ${ }^{3}$ Health Quality Ontario predicted that the average per-patient cost for IOLs in cataract surgery would quadruple (from $\$ 304$ to $\$ 1255$ ) if special-feature IOLs were covered by the Ontario Health Insurance Plan (OHIP). Given the current fiscal constraints on health care funding and the
Competing interests: Ike Ahmed has received consultant or speaker fees from Alcon, Abbott Medical Optics, Bausch \& Lomb and Zeiss. No other competing interests were declared.

This article has been peer reviewed.

Correspondence to: Chryssa McAlister, chryssa.mcalister@utoronto.ca

CMAJ 2015. DOI:10.1503 /cmaj.141601

\section{KEY POINTS}

- In Canada, patients with cataracts can have their vision restored by publicly insured, high-quality and safe surgery without paying extra money out-of-pocket.

- Noninsured services related to refractive correction during cataract surgery are optional for Canadian patients and may include specialized diagnostics, procedures and special-feature intraocular lenses.

- Noninsured testing may provide patients with more predictable and customized refractive results, reduced dependence on corrective lenses and enhanced quality of vision.

- Ethical challenges relating to patient vulnerabilities, conflicts of interest, informed consent, transparency and fairness are important to consider when discussing noninsured services provided alongside insured cataract surgery with patients. 
historical exclusion of refractive correction from provincial health coverage, the mixed model of noninsured and insured services being provided together is likely to continue in Ontario. ${ }^{4}$

\section{How does the system currently work in Canada?}

Physicians performing medically necessary cataract surgery in all settings are paid the same surgical fees by publicly funded insurance plans. Hospitals and independent health facilities are paid a facility fee by the province or territory to cover the operational costs of cataract surgery. Private surgical centres do not receive facility funding and are expected to cover costs such as surgical equipment, facility fees and disposable items in their overhead expenses, and they are prohibited in the Canada Health Act from charging costs to patients when performing medically necessary cataract surgery. ${ }^{5}$

Hospitals, independent health facilities and private surgical centres may all provide noninsured refractive services to patients undergoing publicly funded cataract surgery. In all surgical settings, out-of-pocket fees are charged to patients interested in these noninsured advanced technology options, which further complicates the reimbursement model for cataract surgery. Fees for these services can vary by provider and by surgical facility, and are expected to be a "reasonable" amount by the provincial or territorial regulatory body. ${ }^{6}$

Preoperative noninsured testing is not provided at publicly funded hospitals and takes place in private offices, usually after publicly funded consultations. This testing includes optical biometry, which potentially provides enhanced refractive precision with IOL selection compared with publicly insured ultrasound biometry. ${ }^{7}$ In most provinces and territories, optical biometry is a noninsured service, and surgeons charge patients directly for this before the operation. The decision to select noninsured special-feature IOLs, astigmatism management and/or specialized surgical diagnostics is made in physician offices. In Ontario, payment for special-feature IOLs and specialized surgical diagnostics is made to the hospital or surgical centre. These refractive services are provided at surgical facilities concurrent with the publicly funded portion of the cataract procedure.

\section{What are the unique ethical challenges?}

On the surface, noninsured cataract services seem very similar to noninsured laser refractive surgery, such as laser-assisted in-situ keratomile- usis (LASIK), an innovation that uses a laser to reshape the surface of the cornea to help patients see properly without corrective lenses. In Canada, LASIK has always been covered by private insurance plans only, under a model of fair market value. Both LASIK and noninsured refractive components of cataract surgery are considered convenience items, much like cosmetic surgery, and they are optional for patients who seek enhanced visual quality and reduced dependence on corrective lenses. However, important differences exist in the vulnerabilities of these two distinct patient populations.

Patients considering LASIK are usually young and informed about the procedure before seeking consultation, with an awareness of underlying costs. In contrast, patients with cataracts are usually older and come under the care of the ophthalmologist through referral from primary care providers. Patients with cataracts have vulnerabilities related to their condition, such as visual impairment and potential loss of independence, which may complicate the process of informed consent. What is a fair cost for a noninsured service may not be as readily apparent to the patient undergoing cataract surgery as it is to the LASIK client with only refractive error. Patients referred for cataract surgery often have no prior knowledge of noninsured options or the associated fees, and the concept of combining insured and noninsured services can be confusing. The diversity of the Canadian population may present additional vulnerabilities in patients with cataracts, such as language and cultural barriers. Finally, research on surgical innovation suggests many patients will often perceive new technology to be inherently better, even without supportive evidence. ${ }^{8}$

Ophthalmologists providing noninsured services should consider the potential harm of financial burden on a vulnerable patient with cataracts. Patients who misunderstand the optional nature of noninsured services may make substantial sacrifices to pay for cataract surgery. Alternatively, they may decide to postpone or forgo surgery until they can afford the noninsured costs, which will leave them to suffer unnecessarily for longer with correctable impaired vision.

Patients with cataracts have a visual impairment for which the only treatment and cure is surgery. This temporary impairment can prevent them from functioning, driving, working, and supporting themselves and their families. Those who lose their licence to drive can be desperate to have surgery if driving is required for their livelihood. Furthermore, patients with visual impairment have higher rates of depression and 
worse scores on quality-of-life scales than agematched controls. ${ }^{9,10}$ Canadian law recognizes patient vulnerabilities in the patient-physician relationship and emphasizes the physician's fiduciary obligation to avoid conflict of duty and self-interest. ${ }^{11}$

A 2014 report by the Ontario Health Coalition alleges that some ophthalmologist offices provided misinformation regarding medically necessary and unnecessary options, charged high fees to patients, and violated the Canada Health Act through extra billing and queuejumping. ${ }^{12}$ Although the report oversimplifies the issues and is based on methodologically flawed research, it raises important ethical considerations regarding the combining of insured and noninsured services. Extra billing and queue-jumping are legally prohibited in the Commitment to the Future of Medicare $\mathrm{Act}^{13}$ and violate the 2004 Canadian Medical Association (CMA) Code of Ethics, a fundamental responsibility of which is to "consider first the well-being of the patient." 14 Trust is the essence of this fiduciary duty and is especially important when the power imbalance is increased by patient vulnerabilities.

Ophthalmologists providing noninsured services are faced with several financial conflicts inherent to expensive technologies. They may own the equipment that provides noninsured diagnostics, and/or they may have expensive overhead from running a private operating room. They are also limited by law in what they can charge patients and must charge reasonable fees for noninsured extra services independent of overhead costs. ${ }^{5,6}$ Furthermore, Ontario hospitals, surgical centres and physicians are prohibited by law from charging patients profit-incurring fees for any special-feature IOLs. ${ }^{15}$ These conflicts demand deliberation and evaluation to ensure they are resolved in the best interest of the patient.

The College of Physicians and Surgeons of Ontario, in a policy statement on block fees and uninsured services, advises the physician to "put the needs of the patient first" when resolving the conflicts of interest inherent to the provision of noninsured options. ${ }^{6}$ A statement from the Canadian Ophthalmological Society provides guidance regarding service fees ${ }^{16}$ but does not make reference to ethical values or considerations. The CMA Code of Ethics suggests that physicians should "consider both the nature of the service provided and the ability of the patient to pay" in determining fees for noninsured services and that charges should not be onerous or exaggerated. ${ }^{14}$ Canadian patients rely on physicians to present fair and reasonable management options for both insured and noninsured services.
Some practices are clear conflicts of interest and are prohibited by law. ${ }^{17}$ An example is feesplitting, otherwise known as offering kickbacks, in which a referring practitioner is compensated for providing a patient referral. Fee-splitting has long been established as unethical ${ }^{18}$ and not compatible with a physician's fiduciary duty owing to its inherent conflict with patient interests and demonstrated effect on clinical judgment. ${ }^{19-21}$ Despite this, anecdotal reports of kickbacks in ophthalmology continue in Canada ${ }^{21}$ through direct payments to referring optometrists - sometimes under the guise of patient education on noninsured services - and unlinked payments from manufacturing companies to ophthalmologists for implanting special-feature IOLs. Fee-splitting cannot be rectified by disclosure and violates the moral foundation of the patient-physician relationship. ${ }^{21}$

\section{Our duty to the patient}

Patients have the right to be informed of all choices available, and ophthalmologists must provide them with "the information they need to make informed decisions about their medical care." ${ }^{14}$ Simply avoiding the discussion, or not providing noninsured options to patients, does not give patients adequate knowledge to make informed decisions. Patients who wish to enhance their refractive result at the time of cataract surgery and who make a properly informed decision should have full access to noninsured options. At the same time, physicians should not misrepresent medically necessary and unnecessary services when both insured and noninsured options exist. This is particularly true for patients with cataracts, given the potential added vulnerabilities of these patients.

Ophthalmologists must take care not to use biased language, such as "premium" for specialfeature IOLs or "old-fashioned" for insured standard testing, ${ }^{12}$ and must ensure that patients understand that all noninsured options are convenience items. Furthermore, delegation of the consent discussion to nonmedical staff may not be satisfactory. Physicians must recognize that nonmedical employees involved in counselling patients on noninsured options may not appreciate the vulnerabilities of the patient or the influence value-based language has on the patient's understanding of options. Ultimately, it is the physician's responsibility to ensure that the patient is fully informed.

Recommendations are needed to ensure fairness and transparency in the delivery of insured and noninsured cataract services, and ophthalmologists need to lead this conversation in clini- 
cal practice, in public, with provincial and territorial regulators, and in academia. Canadian ophthalmologists should inform their patients that publicly insured cataract surgery involves state-of-the-art phacoemulsification technology with high-quality intraocular lenses that will restore their visual acuity. All patients should be made aware of, and may choose, optional, nonmedically necessary specialty diagnostics, lenses and lasers, to potentially enhance visual quality, reduce dependence on corrective lenses, and/or reduce their prescription strength.

The public must be made aware of the dilemmas surrounding mixed insured and noninsured services. We have developed an online patient handout with the Eye Physicians and Surgeons of Ontario (www.epso.ca) outlining the difference between publicly insured and noninsured cataract services, with the goal of empowering patients and primary care providers with information in a more transparent, accessible and comprehensive manner. ${ }^{22}$ Referring providers play a role in educating and advocating for patients who are contemplating cataract surgery. Public input will ultimately help elucidate the most effective way of reaching Ontarians.

Ophthalmology organizations in Canada need to address and better educate their membership through guidelines, codes of ethics and practice standards that reflect the current ethical struggles in providing cataract services. The Eye Physicians and Surgeons of Ontario created a specialty-specific code of ethics in 2014 to "ensure that guidelines exist on issues unique to the practice of ophthalmology." ${ }^{23}$ Education has the potential to empower the patient and help surgeons in navigating the ethical complexities of providing noninsured services during insured cataract surgery.

\section{References}

1. Minassian DC, Rosen P, Dart JK, et al. Extracapsular cataract extraction compared with small incision surgery by phacoemulsification: a randomized trial. Br J Ophthalmol 2001;85:822-9.

2. Horn JD. Status of toric intraocular lenses. Curr Opin Ophthalmol 2007; 18:58-61.

3. Health Quality Ontario. Intraocular lenses for the treatment of age-related cataracts. Ont Health Technol Assess Ser 2009;9:1-62.

4. Butler D. Rising cost creating 'extreme financial constraints' for funding new drugs in Ontario. Ottawa Citizen 2014 Apr. 8.
5. Canada Health Act Annual Report 2010-2011. Ottawa: Health Canada; 2012. Available: www.hc-sc.gc.ca/hcs-sss/pubs/cha -lcs/2011-cha-lcs-ar-ra/index-eng.php (accessed 2014 Nov. 9).

6. Block fees and uninsured services. Toronto: College of Physicians and Surgeons of Ontario; 2010. Available: www.cpso.on.ca /policies-publications/policy/block-fees-and-uninsured-services (accessed 2014 Nov. 9).

7. Rajan MS, Keilhorn I, Bell JA. Partial coherence laser interferometry vs conventional ultrasound biometry in intraocular lens power calculations. Eye (Lond) 2002;16:552-6.

8. American College of Surgeons. Statement on issues to be considered before new surgical technology is applied to the care of patients. Committee on Emerging Surgical Technology and Education, American College of Surgeons. Bull Am Coll Surg 1995; 80:46-7.

9. Renaud J, Bedard E. Depression in the elderly with visual impairment and its associations with quality of life. Clin Interv Aging 2013;8:931-43.

10. Rees G, Xie J, Holloway EE, et al. Identifying distinct risk factors for vision-specific distress and depressive symptoms in people with vision impairment. Invest Ophthalmol Vis Sci 2013;54:7431-8.

11. Norberg v. Wynrib, [1992] 2 SCR 226.

12. For health or wealth? The evidence regarding private clinics and user fees in Ontario. Toronto: Ontario Health Coalition; 2014. Available: www.ontariohealthcoalition.ca/wp-content/ uploads/March-25-2014-Private-Health-Clinics-Full-Report.pdf (accessed 2014 Dec. 1).

13. Commitment to the Future of Medicare Act. Toronto: Government of Ontario; 2004. Available: www.e-laws.gov.on.ca/html/statutes/ english/elaws_statutes_04c05_e.htm (accessed 2014 Nov. 11).

14. CMA Code of Ethics (Update 2004). Ottawa: Canadian Medical Association; 2004. Available: http://policybase.cma.ca/dbtw -wpd/PolicyPDF/PD04-06.pdf (accessed 2014 Nov. 11).

15. Medicine Act, 1991: Ontario Regulation 114/94. Toronto: Government of Ontario; 1991. Available: www.e-laws.gov.on.ca/htm /regs/english/elaws_regs_940114_e.htm (accessed 2014 Dec. 1).

16. COS statement on values for uninsured services in Canada. Ottawa: Canadian Ophthalmological Society; 2010. Available: www.cos-sco.ca/wp-content/uploads/2012/09/Uninsured-services _COSstatement_Apr2010.pdf (accessed 2014 Nov. 15).

17. Medicine Act, Ontario Regulation 856/93 Toronto: Government of Ontario; 1991. Available: www.e-laws.gov.on.ca/html/regs/ english/elaws_regs_930856_e.htm (accessed 2014 Dec. 1).

18. Revised Code of Ethics adopted by the Canadian Medical Association June 21, 1938. Toronto: The Canadian Medical Association; 1939:30.

19. Litman M. Self-referral and kickbacks: fiduciary law and the regulation of "trafficking in patients." CMAJ 2004;170:1119-20.

20. Choudhry S, Choudhry NK, Brown AD. Unregulated private market for health care in Canada? Rules of professional misconduct, physician kickbacks and physician self-referral. CMAJ 2004;170:1115-8

21. Levin AV, Ganesh A, Al-Busaidi A. Fee splitting in ophthalmology. Can J Ophthalmol 2011;46:21-7.

22. Cataract surgery in Ontario patient handout 2015. Toronto: Eye Physicians and Surgeons of Ontario; 2015. Available: www.epso.ca/info-for-patients/eye-conditions/cataract/cataract -surgery-in-ontario (accessed 2015 May 20).

23. Code of ethics of the Eye Physicians and Surgeons of Ontario. Toronto: Eye Physicians and Surgeons of Ontario; 2014. Available: www.epso.ca/what_s-new_/code-of-ethics (accessed 2015 May 20).

Affiliation: Department of Ophthalmology and Vision Sciences, University of Toronto, Toronto, Ont.

Contributors: Both of the authors wrote the article, approved the version to be published and agreed to act as guarantors of the work. 